\title{
Reemplazo valvular aórtico transcatéter (RVAT): manejo perioperatorio y consideraciones anestésicas
}

\author{
Transcathether aortic valve replacement (TAVR): \\ perioperative management and anesthetic considerations
}

Maximiliano Zamora H. ${ }^{1}$, Katia González C. ${ }^{2}$, Evelyn Borchert B. ${ }^{3}$, Guillermo Lema F. 4,5

\begin{abstract}
The trans-catheter aortic valve replacement (TAVR) is becoming more frequent and, therefore, there is need for a proper perioperative management. The new devices have reduced the complications and therefore increased the indications of this technique, even in patients with low surgical risk. It is necessary to know the procedure and the types of valves available, the most frequent access is transfemoral and the devices are usually separated in ballon-expandable and self-expanding valves. There is recent evidence that would indicate some benefits of using sedation as the anesthetic technique, however, the choice should be based on each individual patient, the center and the experience of each anesthesiologist in charge. Although TAVR is much safer today, there are multiple complications reported, such as vascular lesions, paravalvular leakage, neurological complications, among others. An adequate knowledge and monitoring of them will allow us to reduce them in the perioperative period.
\end{abstract}

\author{
Key words: \\ Aortic stenosis, \\ surgical aortic valve \\ replacement, \\ trans-catheter aortic \\ valve replacement, \\ ballon-expandable, \\ self-expanding
}

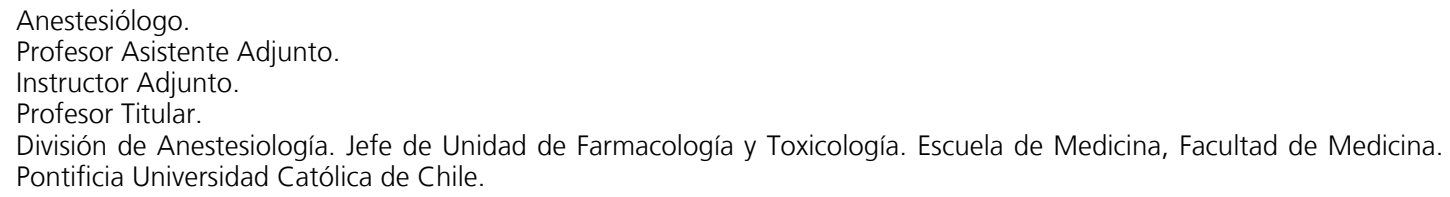

\section{ORCID}

0000-0003-4895-7349

\section{Correspondencia:}

Maximiliano Zamora $\mathrm{H}$.

mszamora@uc.cl 


\section{RESUMEN}

Cada vez es más frecuente el reemplazo valvular aórtico trans-catéter (RVAT) y, por ende, la necesidad de su adecuado manejo perioperatorio. Los nuevos dispositivos han disminuido sus complicaciones y, por lo tanto, aumentado su indicación, incluso en pacientes de bajo riesgo quirúrgico. Es necesario conocer la forma en que se realiza el procedimiento, tipos de válvulas disponibles, accesos más frecuentes y los tipos de dispositivos. Existe evidencia reciente que indicaría algunos beneficios de utilizar sedación como técnica anestésica, sin embargo, la elección se debe basar en cada paciente en particular, el centro y la experiencia de cada anestesiólogo a cargo. Si bien el RVAT hoy es mucho más seguro, existen complicaciones, entre las que se encuentran lesiones vasculares, leak paravalvular, complicaciones neurológicas, tamponamiento, entre otras. Un adecuado conocimiento y monitorización de ellas nos permitirá reducirlas en el perioperatorio.

\author{
Palabras clave: \\ Estenosis aórtica, \\ reemplazo valvular \\ aórtico quirúrgico, \\ reemplazo valvular \\ aórtico transcatéter, \\ balón expandible, \\ autoexpandible
}

\section{Introducción}

Le

a estenosis aórtica es la valvulopatía más frecuente y esta incrementa su incidencia con la edad[1]. - Se espera que la incidencia y complejidad de pacientes con esta enfermedad también aumente con el tiempo, por el envejecimiento de la población.

El manejo estándar de todo paciente con estenosis aórtica severa sintomática correspondió durante mucho tiempo al reemplazo valvular quirúrgico, ya sea con prótesis biológica o mecánica. Esta técnica es bien conocida y presenta excelentes resultados[2]. A pesar de lo anterior, las características de este tipo de pacientes, como ya se mencionó, cada vez más añosos y con mayor número de comorbilidades, han determinado que surjan otras técnicas menos invasivas, con el fin de disminuir el elevado riesgo que la cirugía, que incluye circulación extracorpórea y todas las complicaciones relacionadas a un procedimiento abierto mayor, podría tener sobre estos pacientes. Es por lo anterior que, desde el año 2002, año en el cual se realizó el primer reemplazo valvular aórtico transcatéter (RVAT)[3], el manejo de esta patología ha sufrido cambios considerables, con muchos estudios actuales y guías clínicas apuntando a que esta sería una alternativa adecuada en pacientes de riesgo prohibitivo o elevado (recomendación clase I, evidencia nivel A) y de riesgo intermedio (recomendación clase lla)[4]. Incluso, estudios más recientes aprueban su uso en pacientes de riesgo bajo[5]. En si misma, esta técnica ha ido avanzando a pasos extremadamente rápidos, los dispositivos actuales cuentan cada vez con mayor seguridad y menores efectos adversos, lo que ha aumentado aún más su popularidad.
Es por lo anterior, y teniendo en cuenta que con mayor frecuencia nos veremos enfrentados a la resolución de esta patología por medio del RVAT, es necesario para los anestesiólogos conocer sobre el adecuado manejo perioperatorio de este tipo de pacientes, conocer la técnica y sus posibles complicaciones, saber seleccionar la técnica anestésica según cada tipo de paciente en particular, entre otros temas, que se presentarán en la siguiente revisión.

\section{Indicación de RVAT}

Como se mencionó previamente, en los últimos años el RVAT ha adquirido cada vez mayor aprobación como técnica de elección en el manejo de la estenosis aórtica severa sintomática. Lo anterior corresponde a un "cambio de paradigma" al manejo tradicional de la enfermedad, que correspondía al reemplazo valvular aórtico quirúrgico. A modo de ejemplo, en las guías de la AHA/ACC de manejo de valvulopatías del año 2014, se reconocía al RVAT como la alternativa de preferencia en pacientes con riesgo quirúrgico prohibitivo (clase 1 , nivel de evidencia $\mathrm{B}$ ) y como alternativa a la cirugía en pacientes con riesgo quirúrgico alto (clase lla, nivel de evidencia B). En las guías actualizadas del año 2017, se recomienda RVAT en pacientes de riesgo prohibitivo y alto, como recomendación clase 1, nivel de evidencia A[4]. Lo anterior, se basa principalmente en el estudio PARTNER 1[6], un ensayo clínico randomizado, multicéntrico, que incluyó a 699 pacientes con estenosis aórtica severa sintomática y con riesgo quirúrgico prohibitivo o alto (definido como un riesgo de muerte operatorio mayor o igual al 15\%), comparando los resultados 
del RVAT y la resolución quirúrgica. En el año 2015 se publicaron los resultados de 5 años de seguimiento, aquí se documentó que el riesgo de muerte a 5 años en el caso del RVAT era $67,8 \%$, comparado con $62,4 \%$ en el grupo quirúrgico $(p=0,76)$, por lo que serían alternativas comparables en cuanto a mortalidad. Tampoco se detectaron diferencias en otros resultados como mortalidad por causa cardiovascular, resultados funcionales, mejoría en función valvular, accidente vascular encefálico (AVE) o necesidad de readmisión hospitalaria. Si bien el riesgo de AVE periprocedimiento (a 30 días) es mayor con RVAT que con cirugía $(5,5 \%$ contra $2,4 \%$, respectivamente, $p$ $=0,04)$, a 5 años estas diferencias desaparecen. Otra rama del estudio PARTNER 1[7], comparó los pacientes que se consideraban "inoperables" o no candidatos a cirugía, comparando sus resultados con RVAT y el tratamiento estándar médico, que podía incluir valvuloplatía con balón, demostrando que el RVAT era más beneficioso en este tipo de pacientes. Se debe tener en consideración, que este estudio uso la prótesis Edwards balón expandible, desde entonces estos dispositivos han sido reemplazados por otros de segunda y tercera generación, que utilizan vainas más pequeñas (resultante en menos complicaciones vasculares), poseen la habilidad para sellar leaks para valvulares y otras características más favorables[8], que han determinado mejores resultados en estudios más recientes.

También se han hecho más avances en cuanto a pacientes de menor riesgo, específicamente, el trabajo PARTNER 2[9], también un ensayo clínico randomizado incluyó a 2.032 pacientes con estenosis aórtica severa y riesgo quirúrgico intermedio, también comparando los resultados del RVAT y la cirugía. Aquí los resultados fueron similares al estudio anterior, al comparar ambas técnicas, los resultados en cuanto a mortalidad, AVE e implantación de marcapasos eran similares. El sangrado mayor y aparición de fibrilación auricular eran más comunes en el grupo quirúrgico. Metaanálisis que también utilizan este tipo de pacientes, también confirman estos resultados similares en mortalidad y alteraciones neurológicas[10]-[12]. En consecuencia, a los resultados anteriores, las guías antes mencionadas ahora determinan al RVAT como una alternativa razonable en pacientes con estenosis aórtica severa sintomática y riesgo quirúrgico intermedio, siendo una recomendación clase lla, nivel de evidencia moderado.

Estudios más recientes[5],[13], incluso muestran al RVAT como una alternativa adecuada en pacientes que se consideran de bajo riesgo quirúrgico, con resultados positivos tanto para las válvulas balón expan- dible y autoexpandible. Si bien aún las guías clínicas no adoptan esta nueva evidencia, está claro que la indicación de RVAT ha ido aumentando y probablemente seguirá esta tendencia durante los próximos años, a medida que la técnica y dispositivos se van perfeccionando.

\section{Procedimiento y tipos de válvulas transcatéter}

La forma más frecuente en que se realiza este procedimiento es el acceso transfemoral[14], donde se canula la arteria femoral, con el paso de la válvula de forma retrógrada a través de la aorta ascendente. Sin embargo, existen pacientes en los que la arteria femoral no se encuentra disponible para su canulación, como pacientes con enfermedad arterial periférica oclusiva relevante, arteria con extremada tortuosidad, calcificación marcada, diámetro pequeño u otra enfermedad aórtica (aneurisma o trombo en su interior)[14]. En esos casos, se puede utilizar otro vaso para la canulación arterial y paso retrógrado de la válvula, siendo más frecuente el acceso transaxilar. Si bien es una alternativa adecuada a la vía transfemoral, es más invasiva, ya que requiere de exposición quirúrgica de la arteria subclavia, por lo que no es totalmente percutánea. Otra opción es el paso de la válvula de manera anterógrada, lo que es el llamado acceso transapical[15], en este caso, se requiere una mini toracotomía para acceder al ápex del ventrículo izquierdo, por lo que este procedimiento es generalmente realizado por un cardiocirujano. Otros accesos, como el transaórtico[16] y el transcavo[17], se utilizan con menor frecuencia.

En la actualidad, existen dos tipos de válvulas aprobadas por la FDA (Food and Drug Administration) de los Estados Unidos[18], las cuales corresponden a las utilizadas con mayor frecuencia en los grandes estudios que evalúan los resultados del RVAT. Por una parte, existe la Edwards Sapien System (Edwards Lifesciences Corporation). La válvula Edwards Sapien es balón expandible, posee tres velos, compuesta de pericardio equino unida a un marco de acero inoxidable. Está disponible en tamaños de 23, 26 y 29 mm. La Edwards Sapien XT es la que se utilizó en el estudio PARTNER 1[6],[7], con resultados favorables para pacientes de alto riesgo quirúrgico y pacientes que eran considerados inoperables. La Edwards Sapien 3 implementó características que previenen los leak paravalvulares[19], y es la que fue utilizada en el estudio PARTNER 2 y 3 . Por otro lado, está la CoreVal$\mathrm{ve}^{\circledR}$ Revalving System (Medtronic), que es una válvula autoexpandible, también con tres velos, compuesta de pericardio porcino, existe en diámetros de 23, 26, 
29 y $31 \mathrm{~mm}$. Estas también tienen demostrada efectividad en pacientes de alto riesgo quirúrgico[20],[21]. En cuanto a la comparación entre estos dos tipos de válvulas, un metaanálisis reciente[22], demostró que entre las válvulas balón expandible y autoexpandibles, existía una mortalidad comparable y un riesgo de AVE similar. Sin embargo, en el mismo estudio, las válvulas autoexpandibles, tenían una mayor incidencia de necesidad de implantación de un nuevo marcapasos, insuficiencia aórtica, embolización de la válvula, y necesidad de ocupar más de una válvula, al compararlas con las balón expandibles. Otros estudios demuestran resultados similares, sobre todo confirmando la mayor necesidad de implantación de marcapaso definitivo y mayor estadía hospitalaria, en el caso de las válvulas autoexpandibles[23],[24]. Usualmente, se realiza una valvuloplastía con balón previo al paso de la válvula, para facilitar su paso por el área estenótica, siendo esto no necesario cuando se hace a través de una prótesis estenótica (procedimiento "válvula sobre válvula")[14]. Con las válvulas balón expandibles de Edwards, usualmente se requiere marcapasos ventricular rápido para disminuir la presión arterial y el flujo transvalvular, para lograr reducir el riesgo de movimiento de la válvula hacia la aorta durante su despliegue, por lo que se debe instalar una sonda marcapaso al paciente. En el caso de la CoreValve, al ser más larga, permite un despliegue gradual de la prótesis, sin necesidad de marcapasos[25].

Actualmente, la elección de la válvula tiene que ver con la situación específica del paciente. La válvula autoexpandible se prefiere cuando el ánulo aórtico tiene forma ovalada, el diámetro de la arteria femoral es de 5 a $5,5 \mathrm{~mm}$, o con un ánulo severamente calcificado, donde podría haber mayor riesgo de ruptura. Estas también tienen la habilidad de poder ser reposicionadas, lo que es de gran utilidad cuando hay riesgo alto de obstrucción de los ostium coronarios. En el caso de las válvulas balón expandibles, estas son la única opción en el acceso transapical y se prefieren en casos con aorta ascendente dilatada (más de $43 \mathrm{~mm}$ ) o aorta severamente angulada[26].

\section{Manejo anestésico del RVAT}

Si bien el número de pacientes que ingresan para un RVAT ha aumentado de manera considerable durante los últimos años, aún no existe consenso con respecto a cuál debe ser el manejo anestésico apropiado para este procedimiento, tampoco existen guías clínicas ni recomendaciones relacionadas a esto[27]. Lo que está claro es que, por los avances de esta técnica y las características de los pacientes, muchas ve- ces frágiles y con gran número de comorbilidades, la tendencia actual es llevar a este procedimiento a ser un evento lo menos invasivo posible, muchas veces con menos instrumentos de monitorización invasiva, anestesia local, más una sedación leve, o lo que es llamado en la literatura como "sedación consciente". Tanto en nuestro centro, como en otros citados en la literatura a continuación, los primeros casos de RVAT se realizaron de forma mandataria con anestesia general, monitorización invasiva que incluía línea arterial, catéter venoso central y muchas veces catéter de arteria pulmonar. Hoy en día, el uso de anestesia general solo es mandatorio cuando se planea un acceso quirúrgico (transapical o transaórtico). En cuanto a la monitorización, esta sigue siendo un punto importante en el manejo perioperatorio de estos pacientes, al menos en nuestra institución, todos estos pacientes se monitorizan con presión arterial invasiva, mediante una línea arterial, además de todos los otros elementos básicos de monitorización (saturometría, electrocardiograma con tres derivaciones, capnografía en caso de utilizar anestesia general). El uso de catéter venoso central o catéter de arteria pulmonar ya no es mandatorio y dependerá de las comorbilidades de cada paciente que se presente al procedimiento[28]. Si muchas veces se instala una sonda marcapasos por razones anteriormente expuestas, dependiendo del tipo de válvula a ser implementada.

\section{Elección de la técnica anestésica}

Uno de los temas más relevantes y, últimamente, más estudiados con respecto al RVAT, es la selección de la técnica anestésica. Este procedimiento se ha realizado de manera satisfactoria con anestesia local, anestesia local más sedación o anestesia general. Si bien los primeros casos fueron desarrollados en su totalidad con anestesia general, y con sedación solo en $5 \%$ total de los casos, actualmente, este paradigma está cambiando, reportándose en análisis actuales hasta $40 \%$ de uso de sedación en Estados Unidos[29]. Parece bastante razonable que esté en aumento el uso de sedación en este tipo de pacientes, ya que una técnica menos invasiva podría eventualmente mejorar los resultados en esta población.

Existen múltiples beneficios al seleccionar la sedación como técnica anestésica. Por una parte, no se requiere manejo de la vía aérea, por lo que esto podría disminuir las complicaciones respiratorias en pacientes añosos y frágiles (tipo de paciente que usualmente se presentan a un RVAT). Sin embargo, si bien la sedación no es una contraindicación para el uso de ecocardiograma transesofágico, muchas ve- 
ces se hace difícil utilizarlo en la ausencia de anestesia general y manipulación de la vía aérea[30], lo que podría interferir con la detección de complicaciones tempranas que muchas veces solo se visualizan con esta técnica. También se hace difícil el uso de la sonda marcapasos, por ser mal tolerada en los pacientes despiertos. Además, siempre existe el miedo de movimientos por parte del paciente, en momentos críticos del procedimiento como durante el despliegue de la válvula. Por lo anterior, la anestesia general suele parecer muchas veces la alternativa más "cómoda" para el anestesiólogo.

Al analizar los resultados expuestos por distintos estudios con respecto a cuál sería la técnica anestésica de elección para este tipo de procedimiento, nos enfrentamos con varias limitaciones. Primero, existe un déficit de ensayos clínicos randomizados evaluando el tema. La mayoría de los trabajos descritos son observacionales lo cual puede aumentar el riesgo de sesgos en su formulación. Lo anterior no es un tema menor, si tomamos en consideración que muchas veces en pacientes con más comorbilidades y más frágiles, muchas veces la anestesia general pasa a ser la técnica de elección, al ser la técnica más "segura" o "mejor conocida" por el anestesiólogo, lo que podría llevar a peores resultados en este grupo de pacientes. También se debe tener en consideración los sesgos relacionados al desarrollo y experiencia de esta técnica por cada centro en específico. La anestesia general es con frecuencia la primera técnica que se utiliza cuando este procedimiento está comenzando en una institución, como se dijo previamente, inicialmente la incidencia de sedación era baja, pero actualmente en Europa la mayor parte de los casos se hacen con sedación, hasta en $60 \%$ según algunos reportes[31], lo que indicaría que los centros con mayor experiencia y con más tiempo en esto tienden a utilizar con mayor frecuencia la sedación con anestesia local, quedando en duda entonces, si los mejores resultados que se podría evidenciar en grupos con sedación, serían causados por la técnica anestésica o simplemente una mayor experiencia del centro. Por otro lado, la definición de sedación muchas veces no es clara en distintos estudios, por lo que se confunden términos como cuidados anestesiológicos monitorizados, sedación consciente, entre otros. Además, rara vez se registran cuáles fueron las drogas a ser utilizadas en la sedación y de forma más extraña aun las dosis que se emplearon.

Teniendo lo anterior en consideración, la mayoría de los estudios revela una tasa de éxito alta del procedimiento, ya sea con sedación o con anestesia general en aproximadamente 97\%[32]. Dos de los estudios observacionales de mayor tamaño[32],[33], han mostrado menores índices de mortalidad hospitalaria y temprana en los pacientes en los cuales se utilizó sedación, además de menor estadía hospitalaria. La disminución de la estadía hospitalaria y en la unidad de cuidados intensivos se ha relacionado con disminución importante de los costos en otros estudios[34],[35]. Un metaanálisis del año 2018[36] comparó los resultados de estos pacientes con anestesia general, contra "sedación consciente" con anestesia local. Aquí se mostró una disminución de la mortalidad a 30 días, menor tiempo de procedimiento, tiempo de fluoroscopía, estadía hospitalaria, estadía en la unidad de cuidados intensivos y menor necesidad de soporte inotrópico, sin embargo, otros trabajos de similar metodología no demuestran este efecto sobre la mortalidad[37], pero sí mantienen el efecto beneficioso de la sedación con anestesia local sobre la estadía hospitalaria y en la unidad de cuidados intensivos. En conclusión, la mayor parte de los estudios evaluando las dos técnicas anestésicas muestran una mortalidad a 30 días similar, pero con una consistente disminución de los tiempos hospitalarios y costos. A pesar de lo anterior, la decisión de la técnica anestésica debería ser individualizada según el paciente es por lo anterior que la evaluación previa del anestesiólogo pasa a ser fundamental. Probablemente, en pacientes selectos y cooperadores, la sedación con anestesia local podría otorgar beneficios en cuanto a resultados, incluso en poblaciones especiales, como los pacientes con enfermedad pulmonar obstructiva crónica (EPOC), podría tener aún mejores resultados[38], pero claramente hay condiciones en las cuales uno debería orientarse más por la anestesia general; a modo de ejemplo, pacientes poco cooperadores, obesos o con antecedente de vía aérea difícil, en los cuales una conversión a anestesia general podría ser menos segura y laboriosa[39]. En el caso de requerir acceso quirúrgico abierto para el RVAT, si bien lo clásico ha sido utilizar anestesia general, también existen reportes de éxito con cuidados anestesiológicos monitorizados y bloqueo ilioinguinal[40], por lo que, el uso de anestesia general, no sería mandatorio en estos casos.

Además de lo anterior, siempre hay que estar preparado para una eventual conversión a anestesia general, lo que ocurre en promedio en $6 \%$ de los casos[30], en un estudio[41] se demostró mayor incidencia de efectos adversos como bradipnea, necesidad de maniobras de vía aérea o necesidad de ventilar con bolsa mascarilla en un grupo de pacientes en los que se utilizó sedación para el RVAT, al compararlo con los pacientes que desde un principio se había elegido anestesia general. 
Queda claro entonces, que el rol de la evaluación del anestesiólogo y su rol en pabellón pasa a ser fundamental, ya que podrá hacer una selección individualizada de qué tipo de anestesia proporcionará para cada paciente, además de estar preparado en caso de un eventual paso a anestesia general, si la sedación fue la técnica elegida en un comienzo. Si bien la evidencia actual apunta a que la sedación con anestesia local tendría claros beneficios, la decisión de qué tipo de anestesia se debe proporcionar dependerá, no solo del paciente y sus comorbilidades, sino que también del centro en el que estemos y la experiencia que se ha formado en aquel lugar. Con todo lo anterior expuesto, a la fecha, faltan estudios de calidad que nos permitan hablar de una técnica anestésica única y superior al resto.

\section{Complicaciones RVAT}

Si bien hemos hablado de los múltiples beneficios de esta técnica en comparación a la cirugía tradicional, también hay que tener en cuenta que el RVAT tiene sus propios tipos de complicaciones. Existe isquemia reportada derivada del uso de marcapasos al desplegar la válvula. También riesgo de necesidad de un marcapasos definitivo, lo que es cuatro veces más frecuente con la CoreValve.

Uno de los avances más importantes en relación a las complicaciones en RVAT, ha sido la reducción de las lesiones vasculares. Los sistemas iniciales utilizaban vainas de 22 a 25 French y esto sumado a una menor experiencia de los operadores, era frecuente la incidencia de disección y perforación arterial. Los nuevos productos disponibles son compatibles con vainas mucho más pequeñas, hasta de 18 French[42].

Si bien la incidencia de insuficiencia aórtica leve a moderada es frecuente luego del procedimiento, que esta sea severa solo ocurre en $5 \%$ de los casos[42]. Por lo que, las alteraciones hemodinámicas relacionadas a esto son relativamente infrecuentes. También existen casos de mal posición y embolización de las válvulas, las cuales, si bien son infrecuentes, requieren habitualmente resolución quirúrgica. El despliegue de la válvula también puede precipitar oclusión coronaria, con alteraciones en el balance de aporte y demanda de oxígeno miocárdico y posterior isquemia. Para evitar esta complicación, es esencial la buena preparación con una adecuada aortografía previa y uso de ETE periprocedimiento. El tamponamiento cardiaco es una complicación infrecuente, pero que puede ser detectado de manera precoz también con el uso de ETE. También existe una reducción importante de la incidencia de leak paravalvular con los nuevos dispositivos y con mayor frecuencia existe experiencia para resolverlos, ya sea con una nueva válvula o Amplatzer.

Finalmente, en cuanto a las complicaciones neurológicas, si bien la incidencia de accidente cerebrovascular (AVE) con manifestaciones clínicas es baja (alrededor del 2\%), hasta en $80 \%$ de los pacientes se encuentran lesiones a nivel de resonancia nuclear magnética[43]. Estas lesiones, si bien no se manifiestan de forma inmediata como déficit neurológico, pueden predisponer a déficit cognitivo tiempo después del procedimiento. Actualmente se cuenta con sistemas de protección antiembólica que se utilizan durante el procedimiento[44], que si bien son efectivas, su costo y falta de entrenamiento en su uso muchas veces limitan su expansión.

\section{Conclusiones}

Con los avances en la técnica de RVAT, cada vez con mayor frecuencia nos veremos enfrentados a este procedimiento como anestesiólogos, el cual tiene un grupo de complicaciones en particular y un manejo anestésico específico. El avance en los dispositivos ha permitido que actualmente la indicación de RVAT sea mucho más amplia, incluso en pacientes de bajo riesgo quirúrgico según últimos estudios. El acceso más frecuente es transfemoral, pero existen otros como transaxilar y transapical. Es necesario conocer los distintos tipos de válvulas disponibles, ya que tienen características particulares que pueden determinar cambios en el manejo anestésico. La elección de la técnica anestésica debe ser evaluada en cada paciente en particular, si bien la evidencia actual apunta a un beneficio al utilizar sedación, comparándola con anestesia general. En cualquier caso, la presencia de un anestesiólogo con experiencia en el procedimiento en pabellón, será beneficioso para el paciente. El RVAT no está exento de complicaciones, como lesiones vasculares, leak paravalvular, complicaciones neurológicas, tamponamiento, entre otras. Si bien estas son menos frecuentes relacionado al avance de esta técnica y dispositivos más seguros, aún hay medidas que se pueden tomar para disminuir la incidencia de estas. 


\section{Referencias}

1. Rashedi N, Otto CM. Aortic Stenosis: Changing Disease Concepts. J Cardiovasc Ultrasound. 2015 Jun;23(2):59-69. https://doi. org/10.4250/jcu.2015.23.2.59 PMID:26140146

2. Fassl J, Augoustides JG. Transcatheter aortic valve implantation-part 1: development and status of the procedure. J Cardiothorac Vasc Anesth. 2010 Jun;24(3):498-505. https://doi. org/10.1053/j.jvca.2009.06.011 PMID:19942452

3. Shreenivas S, Kaneko T, Tang $\mathrm{GH}$. Predicting the future of TAVR: an obituary to open aortic valve replacement? Curr Opin Cardiol. 2019 Mar;34(2):11223. https://doi.org/10.1097/ HCO.0000000000000609 PMID:30640745

4. Matiasz R, Rigolin VH. 2017 Focused Update for Management of Patients With Valvular Heart Disease: Summary of New Recommendations. J Am Heart Assoc. 2018;7(1):e007596. https://doi.org/10.1161/ JAHA.117.007596.

5. Mack MJ, Leon MB, Thourani VH, Makkar R, Kodali SK, Russo M, et al.; PARTNER 3 Investigators. Transcatheter Aortic-Valve Replacement with a Balloon-Expandable Valve in Low-Risk Patients. N Engl J Med. 2019 May;380(18):1695-705. https://doi.org/10.1056/NEJMoa1814052 PMID:30883058

6. Mack MJ, Leon MB, Smith CR, Miller DC, Moses JW, Tuzcu EM, et al.; PARTNER 1 trial investigators. 5-year outcomes of transcatheter aortic valve replacement or surgical aortic valve replacement for high surgical risk patients with aortic stenosis (PARTNER 1): a randomised controlled trial. Lancet.
2015 Jun;385(9986):2477-84.

https://doi.org/10.1016/

s0140-6736(15)60308-7

PMID:25788234

7. Kapadia SR, Leon MB, Makkar RR, Tuzcu EM, Svensson LG, Kodali $S$, et al.; PARTNER trial investigators. 5-year outcomes of transcatheter aortic valve replacement compared with standard treatment for patients with inoperable aortic stenosis (PARTNER 1): a randomised controlled trial. Lancet. 2015 Jun;385(9986):2485-91. https://doi.org/10.1016/ S0140-6736(15)60290-2 PMID:25788231

8. Kappetein AP. PARTNERs in the future of surgical aortic valve replacement. Lancet. 2015 Jun;385(9986):2439-41. https://doi.org/10.1016/ S0140-6736(15)60568-2 PMID:25788233

9. Leon MB, Smith CR, Mack MJ, Makkar RR, Svensson LG, Kodali SK, et al.; PARTNER 2 Investigators. Transcatheter or Surgical Aortic-Valve Replacement in Intermediate-Risk Patients. N Engl J Med. 2016 Apr;374(17):160920. https://doi.org/10.1056/NEJMoa1514616 PMID:27040324

10. Elmaraezy A, Ismail A, Abushouk Al, Eltoomy M, Saad S, Negida $A$, et al. Efficacy and safety of transcatheter aortic valve replacement in aortic stenosis patients at low to moderate surgical risk: a comprehensive meta-analysis. BMC Cardiovasc Disord. 2017 Aug;17(1):234. https://doi. org/10.1186/s12872-017-06681 PMID:28836953

11. Garg A, Rao SV, Visveswaran G, Agrawal S, Sharma A, Garg $L$, et al. Transcatheter Aortic Valve Replacement Versus Surgical Valve Replacement in Low-Intermediate Surgical Risk Patients: A Systematic Review and Meta-Analysis. J Invasive
Cardiol. 2017 Jun;29(6):209-16. PMID:28570236

12. Arora $S, M i s e n h e i m e r ~ J A$, Jones W, Bahekar A, Caughey M, Ramm CJ, et al. Transcatheter versus surgical aortic valve replacement in intermediate risk patients: a meta-analysis. Cardiovasc Diagn Ther. 2016 Jun;6(3):241-9. https://doi. org/10.21037/cdt.2016.03.04 PMID:27280087

13. Popma JJ, Deeb GM, Yakubov SJ, Mumtaz M, Gada H, O'Hair D, et al.; Evolut Low Risk Trial Investigators. Transcatheter Aortic-Valve Replacement with a Self-Expanding Valve in LowRisk Patients. N Engl J Med. 2019 May;380(18):1706-15. https://doi.org/10.1056/NEJMoa1816885 PMID:30883053

14. Klein AA, Skubas NJ, Ender J. Controversies and complications in the perioperative management of transcatheter aortic valve replacement. Anesth Analg. 2014 Oct;119(4):784-98. https://doi.org/10.1213/ ANE.0000000000000400 PMID:25232691

15. Lichtenstein SV, Cheung A, Ye J, Thompson CR, Carere RG, Pasupati S, et al. Transapical transcatheter aortic valve implantation in humans: initial clinical experience. Circulation. 2006 Aug;114(6):591-6. https://doi. org/10.1161/CIRCULATIONAHA.106.632927 PMID:16880325

16. O' Sullivan KE, Hurley ET, Segurado R, Sugrue D, Hurley JP; KE OS. Transaortic TAVI Is a Valid Alternative to Transapical Approach. J Card Surg. 2015 May;30(5):381-90. https:// doi.org/10.1111/jocs. 12527 PMID:25756746

17. Lederman RJ, O’Neill WW, Greenbaum AB. Transcaval access for TAVR across a polyester aortic graft. Catheter Cardiovasc Interv. 2015 Jun;85(7):1270-3. 
https://doi.org/10.1002/

ccd.25781 PMID:25510917

18. Arora S, Misenheimer JA, Ramaraj R. Transcatheter Aortic Valve Replacement: Comprehensive Review and Present Status. Tex Heart Inst J. 2017 Feb;44(1):2938. https://doi.org/10.14503/ THIJ-16-5852 PMID:28265210

19. Ribeiro HB, Urena M, Allende R, Amat-Santos IJ, RodésCabau J. Balloon-expandable prostheses for transcatheter aortic valve replacement. Prog Cardiovasc Dis. 2014 MayJun;56(6):583-95. https://doi. org/10.1016/j.pcad.2014.02.001 PMID:24838134

20. Adams DH, Popma JJ, Reardon MJ, Yakubov SJ, Coselli JS, Deeb GM, et al.; U.S. CoreValve Clinical Investigators. Transcatheter aortic-valve replacement with a self-expanding prosthesis. N Engl J Med. 2014 May;370(19):17908. https://doi.org/10.1056/NEJMoa1400590 PMID:24678937

21. Barbanti M, Petronio AS, Ettori F, Latib A, Bedogni F, De Marco F, et al. 5-Year Outcomes After Transcatheter Aortic Valve Implantation With CoreValve Prosthesis. JACC Cardiovasc Interv. 2015 Jul;8(8):1084-91. https://doi. org/10.1016/j.jcin.2015.03.024 PMID:26117458

22. Agarwal $S$, Parashar $A$, Kumbhani DJ, Svensson LG, Krishnaswamy A, Tuzcu EM, et al. Comparative meta-analysis of balloon-expandable and self-expandable valves for transcatheter aortic valve replacement. Int J Cardiol. 2015 Oct;197:87-97. https://doi.org/10.1016/j. ijcard.2015.06.002 PMID:26117654

23. Sathananthan J, Ding $L, Y u$ M, Catlin B, Chan A, Charania J, et al. Implications of Transcatheter Heart Valve Selection on Early and Late
Pacemaker Rate and on Length of Stay. Can J Cardiol. 2018 Sep;34(9):1165-73. https://doi. org/10.1016/j.cjca.2018.06.012 PMID:30170672

24. Rogers $T$, Steinvil A, Buchanan $K$, Alraies MC, Koifman E, Gai J, et al. Contemporary transcatheter aortic valve replacement with third-generation balloon-expandable versus self-expanding devices. J Interv Cardiol. 2017 Aug;30(4):356-61. https:// doi.org/10.1111/joic.12389 PMID:28493389

25. Popma JJ, Adams DH, Reardon MJ, Yakubov SJ, Kleiman NS, Heimansohn D, et al.; CoreValve United States Clinical Investigators. Transcatheter aortic valve replacement using a self-expanding bioprosthesis in patients with severe aortic stenosis at extreme risk for surgery. J Am Coll Cardiol. 2014 May;63(19):1972-81. https://doi. org/10.1016/j.jacc.2014.02.556 PMID:24657695

26. Goeddel LA, Abernathy JH, Brady MB. An Anesthesiologist's Guide to the 2017 American College of Cardiology Expert Consensus Decision Pathway for Transcatheter Aortic Valve Replacement in the Management of Adults with Aortic Stenosis. J Cardiothorac Vasc Anesth. 2019 Feb;33(2):263-73. https://doi. org/10.1053/j.jvca.2018.05.006 PMID:29935802

27. Guarracino F, Baldassarri R. The Anesthetic Management of Transcatheter Aortic Valve Implantation. Semin Cardiothorac Vasc Anesth. 2016 Jun;20(2):141-6. https://doi. rg/10.1177/1089253215606220 PMID:26403787

28. Franco A, Gerli C, Ruggeri L, Monaco F. Anaesthetic management of transcatheter aortic valve implantation. Ann Card Anaesth. 2012 Jan-
Mar;15(1):54-63. https://doi. org/10.4103/0971-9784.91484 PMID:22234024

29. Neuburger PJ, Patel PA, Williams MR. Anesthetic Technique for TAVR: More Than Just "Tube" or "No Tube". J Cardiothorac Vasc Anesth. 2018 Apr;32(2):672-4. https://doi. org/10.1053/j.jvca.2017.08.038 PMID:29153930

30. Sato K, Jones PM. Sedation versus general anesthesia for transcatheter aortic valve replacement. J Thorac Dis. 2018 Nov;10(S30 Suppl 30):S3588-94. https://doi.org/10.21037/ jtd.2018.08.89 PMID:30505539

31. Oguri $A$, Yamamoto $M$, Mouillet G, Gilard M, Laskar M, Eltchaninoff $\mathrm{H}$, et al.; FRANCE 2 Registry Investigators. Clinical outcomes and safety of transfemoral aortic valve implantation under general versus local anesthesia: subanalysis of the French Aortic National CoreValve and Edwards 2 registry. Circ Cardiovasc Interv. 2014 Aug;7(4):602-10. https://doi.org/10.1161/CIRCINTERVENTIONS.113.000403 PMID:25006175

32. Husser $O$, Fujita B, Hengstenberg $C$, Frerker $C$, Beckmann A, Möllmann H, et al.; GARY Executive Board. Conscious Sedation Versus General Anesthesia in Transcatheter Aortic Valve Replacement: The German Aortic Valve Registry. JACC Cardiovasc Interv. 2018 Mar;11(6):567-78. https://doi. org/10.1016/j.jcin.2017.12.019 PMID:29566803

33. Hyman MC, Vemulapalli $S$, Szeto WY, Stebbins A, Patel PA, Matsouaka RA, et al. Conscious Sedation Versus General Anesthesia for Transcatheter Aortic Valve Replacement: Insights from the National Cardiovascular Data Registry Society of Thoracic Surgeons/American Co- 
llege of Cardiology Transcatheter Valve Therapy Registry. Circulation. 2017 Nov; 136(22):213240. https://doi.org/10.1161/ CIRCULATIONAHA. 116.026656 PMID:28864443

34. Toppen W, Johansen D, Sareh S, Fernandez J, Satou N, Patel KD, et al. Improved costs and outcomes with conscious sedation vs general anesthesia in TAVR patients: time to wake up? PLoS One. 2017 Apr;12(4):e0173777. https://doi.org/10.1371/journal. pone.0173777 PMID:28379981

35. Babaliaros $V$, Devireddy $C$, Lerakis S, Leonardi R, Iturra SA, Mavromatis $\mathrm{K}$, et al. Comparison of transfemoral transcatheter aortic valve replacement performed in the catheterization laboratory (minimalist approach) versus hybrid operating room (standard approach): outcomes and cost analysis. JACC Cardiovasc Interv. 2014 Aug;7(8):898-904. https://doi. org/10.1016/j.jcin.2014.04.005 PMID:25086843

36. Villablanca PA, Mohananey D, Nikolic K, Bangalore S, Slovut DP, Mathew V, et al. Comparison of local versus general anesthesia in patients undergoing transcatheter aortic valve replacement: A meta-analysis. Catheter Cardiovasc Interv. 2018 Feb;91(2):330-42. https:// doi.org/10.1002/ccd.27207 PMID:28738447

37. Ehret $C$, Rossaint $R$, Foldenauer AC, Stoppe C, Stevanovic A, Dohms K, et al. Is local anaesthe- sia a favourable approach for transcatheter aortic valve implantation? A systematic review and meta-analysis comparing local and general anaesthesia. BMJ Open. 2017 Sep;7(9):e016321. https://doi.org/10.1136/ bmjopen-2017-016321 PMID:28951409

38. Condado JF, Haider MN, Lerakis $\mathrm{S}$, Keegan $\mathrm{P}$, Caughron $\mathrm{H}$, Thourani $\mathrm{VH}$, et al. Does minimalist transfemoral transcatheter aortic valve replacement produce better survival in patients with severe chronic obstructive pulmonary disease? Catheter Cardiovasc Interv. 2017 Mar;89(4):775-80. https://doi.org/10.1002/ ccd.26683 PMID:27862901

39. Pani $S$, Cagino J, Feustel $P$, Musuku SR, Raja A, Bruno N, et al. Patient Selection and Outcomes of Transfemoral Transcatheter Aortic Valve Replacement Performed with Monitored Anesthesia Care Versus General Anesthesia. J Cardiothorac Vasc Anesth. 2017 Dec;31(6):2049-54. https://doi. org/10.1053/j.jvca.2017.04.005 PMID:28911896

40. Bianco V, Gleason TG, Kilic A, Lee JS, Schindler JT, Rauso $L$, et al. Open Surgical Access for Transfemoral TAVR Should Not Be a Contraindication for Conscious Sedation. J Cardiothorac Vasc Anesth. 2019 Jan;33(1):39-44. https://doi. org/10.1053/j.jvca.2018.05.036 PMID:30458980

41. Mayr NP, Hapfelmeier A, Martin
K, Kurz A, van der Starre $P$, Babik $B$, et al. Comparison of sedation and general anaesthesia for transcatheter aortic valve implantation on cerebral oxygen saturation and neurocognitive outcome. Br J Anaesth. 2016 Jan;116(1):90-9. https:// doi.org/10.1093/bja/aev294 PMID:26424178

42. Masson JB, Kovac J, Schuler G, Ye J, Cheung A, Kapadia S, et al. Transcatheter aortic valve implantation: review of the nature, management, and avoidance of procedural complications. JACC Cardiovasc Interv. 2009 Sep;2(9):811-20. https://doi. org/10.1016/j.jcin.2009.07.005 PMID:19778768

43. Pagnesi $M$, Martino EA, Chiarito M, Mangieri A, Jabbour RJ, Van Mieghem NM, et al. Silent cerebral injury after transcatheter aortic valve implantation and the preventive role of embolic protection devices: A systematic review and meta-analysis. Int J Cardiol. 2016 Oct;221:97-106. https://doi. org/10.1016/j.ijcard.2016.06.143 PMID:27400304

44. Seeger J, Virmani R, Romero M, Gonska B, Rottbauer W, Wöhrle J. Significant Differences in Debris Captured by the Sentinel Dual-Filter Cerebral Embolic Protection During Transcatheter Aortic Valve Replacement Among Different Valve Types. JACC Cardiovasc Interv. 2018 Sep;11(17):1683-93. https://doi. org/10.1016/j.jcin.2018.06.018 PMID:30154060 